\title{
Optimality of the Distance Dispersion Fixation Identification Algorithm
}

\author{
Beibin $\mathrm{Li}^{*}$ \\ Child Study Center \\ Yale School of Medicine \\ Yale University
}

\author{
Quan Wang ${ }^{\dagger}$ \\ Child Study Center \\ Yale School of Medicine \\ Yale University
}

\author{
Laura Boccanfuso \\ Child Study Center \\ Yale School of Medicine \\ Yale University
}

\author{
Frederick Shic ${ }^{\ddagger}$ \\ Child Study Center \\ Yale School of Medicine \\ Yale University
}

\begin{abstract}
Researchers use fixation identification algorithms to parse eye movement trajectories into a series of fixations and saccades, simplifying analyses and providing measures which may relate to cognition. The Distance Dispersion (I-DD) a widely-used elementary fixation identification algorithm. Yet the "optimality" properties of its most popular greedy implementation have not been described. This paper: (1) asks how "optimal" should be defined, and advances maximizing total fixation time and minimizing number of clusters as a definition; (2) asks whether the greedy implementation of I-DD is optimal, and shows that it is when no fixations are rejected for being too short; and (3) we show that when fixation time rejection criterion are enabled, the greedy algorithm is not optimal. We propose an $O\left(n^{2}\right)$ algorithm which is.
\end{abstract}

Keywords: Fixation Identification, Saccade, Greedy Algorithm

Concepts: -Theory of computation $\rightarrow$ Unsupervised learning and clustering; $\bullet$ Computing methodologies $\rightarrow$ Cluster analysis;

\section{Introduction}

People look at an object longer when they are interested in that object. In eye-tracking analysis, we want to identify these fixations from a stream of eye tracking spatial temporal coordinates. The goal of fixation identification is to reduce the complexity of eye-tracking data while maintaining the essential components for cognitive and visual processing analyses [Salvucci and Goldberg 2000]. Moreover, both online and offline eye tracking calibrations are often based on fixations.

There are many popular fixation identification algorithms, and we use the Distance Dispersion Fixation Identification Algorithm (IDD) [Salvucci and Goldberg 2000; Shic et al. 2008] to analyze eyetracking data here. I-DD states that any two points inside the same fixation should be at most $\epsilon^{\circ}$ away from each other. The most used I-DD algorithm is greedy in nature: it begins with the first gaze point, finds the temporally furthest gaze point no more than $\epsilon^{\circ}$ from any prior points, identifies this group as a fixation, and deletes them from dataset. Worst case running time of greedy I-DD algorithm is $O\left(n^{2}\right)$ because of distance matrix calculation. Often researchers set a minimal fixation time rejection threshold so that extremely short fixations, considered to be non-physiological, are rejected and identified as saccades.

\footnotetext{
*e-mail: beibin.li@yale.edu

†e-mail: quan.wang@yale.edu

‡e-mail: frederick.shic@yale.edu

Permission to make digital or hard copies of part or all of this work for personal or classroom use is granted without fee provided that copies are not made or distributed for profit or commercial advantage and that copies bear this notice and the full citation on the first page. Copyrights for thirdparty components of this work must be honored. For all other uses, contact the owner/author(s). (c) 2016 Copyright held by the owner/author(s).

ETRA '16, March 14-17, 2016, Charleston, SC, USA

ISBN: 978-1-4503-4125-7/16/03

DOI: http://dx.doi.org/10.1145/2857491.2888588
}

Despite its straightforward nature, the I-DD algorithm suffers from some ambiguity. Beyond issues related to parameter selection [Shic et al. 2008], it is unclear whether greedy identification of fixation is arbitrary, given space of all possible identifications. In recognition of this issue, we propose two goals for an optimal fixation identification solution:

Goal G1: maximize total fixation time. In theory, a fixation should be identified when the $\epsilon^{\circ}$ criterion is met. A maximal total fixation time criterion is closely aligned with this principle.

Goal G2: minimize the number of fixations. This is a parsimony principle: points should be considered part of the same $\epsilon^{\circ}$-limited cluster whenever possible.

\section{Optimality without Time Rejection}

Is the greedy I-DD algorithm optimal for the two goals G1 and G2 discussed in Section 1? Let's assume minimal fixation time rejection threshold is not used in this section, and we will add this threshold in the next section.

Without time rejection, a saccade is considered as a fixation by itself. The greedy I-DD satisfies G1 because it puts every point in a fixation, and hence total fixation time is equal to the eye tracker recording time. However, does it also satisfy G2? Yes, we can re-articulate the problem and prove this claim.

Problem: Given an array of $n$ gaze points $\left(p_{1}, p_{2}, p_{3}, \ldots, p_{n}\right)$, we want to find the minimum number of fixations (clusters) according to the I-DD definition that cover all of the gaze points.

Algorithm: We will use set $S$ to record all fixations in our algorithm, where set $S$ contains the start points of every fixation. Once we know where a fixation starts, we can know which points are covered by the fixation, because the greedy I-DD will group all of the possible points that the first point can cover.

1. $S=\{\} ; / /$ empty set

2. $i=1$;

3. $S=S \cup\left\{p_{i}\right\}$;

4. Using distance dispersion threshold, find all the consecutive points (i.e. $p_{i}, p_{i+1}, \ldots, p_{i+j}$ ) that can be covered by the same fixation as $p_{i}$.

5. $i=i+j+1$;

6. If $i<n$, Goto Line 3; else, output set $S$.

Claim: The Greedy I-DD described above produces an optimal solution for $\mathrm{G} 2$, i.e. minimum number of fixations.

Proof: Prove by contradiction. Assume optimal solution is $S *$, and $|S *|<|S|$, where $S$ is the output of our algorithm.

Sort all points in $S$ and $S *$ in time order. $S *=$ $\left\{p_{a} *, p_{b} *, p_{c} *, p_{d} *, p_{e} *, \ldots\right\}, S=\left\{p_{a}, p_{b}, p_{c}, p_{d}, p_{e}, \ldots\right\}$.

Without loss of generality, assume the first point differs in $S *$ and $S$ are $p_{d} *$ and $p_{d}$.

It's easy to see $p_{d} *<p_{d}$; otherwise if $p_{d} * \geq p_{d}$, some points between $p_{d} *$ and $p_{d}$ are not covered in $S *$. 
Therefore, $p_{d}$ 's fixation covers more than $p_{d} *$ 's fixation, which means $p_{e} * \leq p_{e}$.

For the same reason, $p_{f} * \leq p_{f}$. $S$ will cover faster than $S *$, and $S *$ is no better than $S$.

The assumption is false. Thus, our algorithm produces the optimal result.

\section{Time Rejection and DP Algorithm}

In real eye-tracking analysis, we would not identify one gaze point as a "fixation"; instead, the point would be regarded as a saccade. We usually reject gaze groups that last less than $100 \mathrm{~ms}$ because most fixations last longer than that [Salvucci and Goldberg 2000]. For eye trackers with constant recording frequencies, minimal time rejection is equivalent to the minimum number of points in a fixation rejection (without considering data lost). So, for simplicity, we will use minimum number of points in a fixation as the threshold in our analysis, and we will calculate the total number of fixation points instead of total fixation time. The algorithm and proof remain the same even if we consider the data lost.

Is the greedy I-DD algorithm still G2 optimal with time rejection threshold? No, because the optimal G2 solution would identify each gaze point as one group and then reject all gaze groups (hence, 0 fixation as result). So, G2 is not rational as a goal for this scenario, hence we want to use G1 as the goal in this case. Then, is the greedy I-DD optimal in terms of G1? The answer is still no, because of the counter-example shown in Figure 1. If we try to compare every possible partition of the gaze data, we can satisfy the G1 optimization, but the run time is exponential to the input data.

The next question is: can we find an algorithm to modify the I-DD and solve this problem "efficiently"? Luckily, the problem is not NP Hard, and here is a $O\left(n^{2}\right)$ time complex and $O(n)$ space complex algorithm that is based on dynamic programming and divide and conquer. We will call it the "Dynamic Programming on Distance Dispersion Fixation Identification Algorithm" (I-DP-DD).

Algorithm Idea: Reject all groups of gazes that have less than $\lambda$ points and identify them as saccades in our algorithm.

Define $f(i)=$ the index of first point that point $p_{i}$ can cover reversely (run the original greedy algorithm in reverse).

Define $S(i)=$ the optimal solution (number of points covered by all fixations) for points $p_{1}, p_{2}, \ldots, p_{i}$.

Then, we have the relationship:

$S(i)=\max _{f(i)-1 \leq j<i}\left[S(j)+\left\{\begin{array}{ll}i-j & \text { if }(i-j \geq \lambda) \\ 0 & \text { otherwise }\end{array}\right]\right.$

The algorithm builds from $f(n)$ to $f(1)$, which takes $O\left(n^{2}\right)$ time. We know $S(i)=0$ for all $i<\lambda$. Then, we can build from $S(1)$ to $f(n)$, where $S(n)$ is the final solution. Building the $S$ array also takes $O\left(n^{2}\right)$ time.

This algorithm is as fast as calculating distance matrix, which also takes $O\left(n^{2}\right)$ time. This dynamic programming improvement for the I-DD may not be the simplest solution, and computer scientists can try to find cleaner algorithms to satisfy G1 in the minimal fixation time rejection scenario.

\section{Conclusion}

Without minimal time rejection and other rejection standards, the greedy I-DD can identify the least number of fixations for eye tracking data. However, with minimal time rejection, the minimal num-

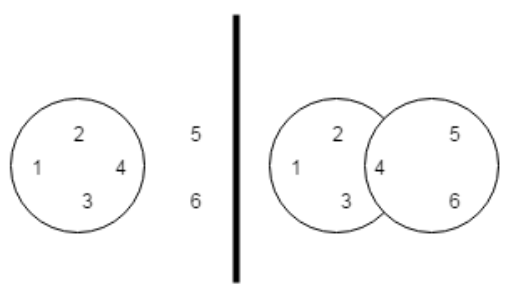

Figure 1: Counter example: Suppose there should be at least 3 points in one fixation. The greedy I-DD will produce the result on the lefthand side, which has one fixation with points 1,2,3, and 4 with a total of 4 points in all fixations. However, the optimal solution on the righthand side has two fixations, where the first fixation has points 1, 2, and 3, and the second fixaiton has points 4, 5, and 6 with a total of 6 points in all fixations.

ber of fixations could not be used as a goal for the distance dispersion algorithm. Instead, Dynamic Programming could be used to improve the I-DD and produce the maximal total fixation time.

\section{Discussion}

Future work can try to improve the greedy I-DD algorithms and apply them to different scenarios. Online and offline fixation identification algorithms can be compared together. Researchers can also use G1 and G2 to analyze other fixation identification algorithms.

As discussed in Introduction, maximizing the total fixation time and minimizing the number of fixations are good goals for fixation identification. Is there any ways to combine them together? We might, for instance, consider G2 is subservient to G1, with a modified criterion that selects the minimal number of fixation after maximizing fixation time. Future studies can work on finding a gold standard or optimal goal for fixation identification.

Most fixation identification algorithms can be classified as either dispersion-based or velocity-based algorithms. Researchers and scientists can try to find other type of algorithms to solve the problem; for instance, Li and his colleagues modified the classical DBSCAN algorithm for fixation identification, combining the advantages from distance and velocity algorithms [Li and Shic 2016]. Future work could focus on the development of new algorithms in conjunction with formalism on optimality criteria.

\section{Acknowledgements}

Thanks to Erin Barney for helpful edits.

Funding was provided by K01 MH104739, R21 MH102572, CTSA UL1 RR024139, R03 MH092618, NIH R01 MH100182, R01 MH087554, U19 MH108206; NSF \#1139078, \#0835767, DOD W81XWH-12-ARP-IDA, and the Nancy Taylor Foundation.

\section{References}

Li, Beibin, W. Q., And ShIC, F. 2016. Modified DBSCAN algorithm on oculomotor fixation identification.

Salvucci, D. D., AND Goldberg, J. H. 2000. Identifying fixations and saccades in eye-tracking protocols. Proceedings of the 2000 symposium on Eye tracking research and applications. $A C M$. (November), 71-78.

Shic, F., Scassellati, B., And Chawarska, K. 2008. The incomplete fixation measure. ETRA 2008, ACM., 111-114. 